\title{
Application of a Machine Learning Algorithm for the Structural Optimization of Circular Arches with Different Cross-Sections
}

\author{
Jonathan Melchiorre, Amedeo Manuello Bertetto*, Giuseppe Carlo Marano \\ Department of Structural Building and Geotechnics, Politecnico di Torino. Corso Duca degli Abruzzi, Torino, Italy \\ Email: *amedeo.manuellobertetto@polito.it
}

How to cite this paper: Melchiorre, J., Bertetto, A.M. and Marano, G.C. (2021) Application of a Machine Learning Algorithm for the Structural Optimization of Circular Arches with Different Cross-Sections. Journal of Applied Mathematics and Physics, 9 , 1159-1170.

https://doi.org/10.4236/jamp.2021.95079

Received: May 3, 2021

Accepted: May 28, 2021

Published: May 31, 2021

\begin{abstract}
Arches are employed for bridges. This particular type of structures, characterized by a very old use tradition, is nowadays, widely exploited because of its strength, resilience, cost-effectiveness and charm. In recent years, a more conscious design approach that focuses on a more proper use of the building materials combined with the increasing of the computational capability of the modern computers, has led the research in the civil engineering field to the study of optimization algorithms applications aimed at the definition of the best design parameters. In this paper, a differential formulation and a MATLAB code for the calculation of the internal stresses in the arch structure are proposed. Then, the application of a machine learning algorithm, the genetic algorithm, for the calculation of the geometrical parameters, that allows to minimize the quantity of material that constitute the arch structures, is implemented. In this phase, the method used to calculate the stresses has been considered as a constraint function to reduce the range of the solutions to the only ones able to bear the design loads with the smallest volume. In particular, some case studies with different cross-sections are reported to prove the validity of the method and to compare the obtained results in terms of optimization effectiveness.
\end{abstract}

\section{Keywords}

Structural Optimization, Genetic Algorithm, Boundary Value Problem, Arch, Volume Minimization

\section{Introduction}

Arch is a very old structural typology due to its effective structural shape that 
can bear the loads in a very efficient way. The main idea behind the arch is to transfer the vertical loads to the supports with a structure that is mainly compressed. The efficiency of the load transfer is strongly related to the arch geometry. Nowadays, arch girders have become again more and more interesting for designers and architects due to their strength, resilience, cost-effectiveness and the possibility of shaping their various forms, influencing, in certain context, the landscape itself [1]. The design of the arch structures goes undoubtedly through their shape. The arches have always been designed looking for the best shape to reduce the internal stress state and, for some applications, for those particular geometries able to ensure, for the greatest number of loading configurations possible, a prevailing compression only condition. In addition, recently, the research of the most performing arch shape together with the study of increasingly detailed calculation methods for the verification of the most diffused geometries, has been accompanied by analysis focused on the varying of the sections along the axis line. Recently some papers investigated the best design parameters concerning the cross-section of arches [2] [3]. Results for optimal shapes of plane-statically determined arches under uniform vertical loads were recently presented [4] [5] [6]. Some papers have been published related to the shaping of the arch axis and shell structures subjected to different loading and constrain conditions [7]-[12]. Concrete arches were studied by Houšt et al. [13], in this case, the best shape configurations were reached in correspondence of the minimization of the maximal stresses. The issue of minimization of the maximal stress over an arch structure in the context of linear elastic thin shell theory was analyzed by [14]. Examples of the determination of the optimum shape of brick masonry arches under dynamic loads by cellular automata were presented by Kumarci et al. [15]. The optimal design of a steel arch bridge by genetic algorithm was considered in recent paper by Park et al. [16]. Shape optimization of concrete open spandrel arch bridges using the simultaneous perturbation stochastic approximation algorithm was studied [17]. An analytical formulation for the calculation of the internal stresses of arches, characterized by different shapes cross-sections is proposed in Section 2. This formulation is based on the solution of a sixth order differential equations system solved respect to the six unknowns represented by the displacements of the arch along the curvilinear abscissa and their first derivative. This formulation, as explained in Section 4, was solved by using the boundary value solver " $b v p 4 c$ " [10] [18] implemented in the software MATLAB [19] by considering the case of a fully restrained arch to define the boundary conditions as in the Section 3. Finally, the solution obtained has been used as constraint function to be implemented in the genetic algorithm [20] [21] that is a machine learning algorithm for the solution of the optimization problem. Nowadays a good design process cannot disregard the evaluation of the structural efficiency aimed to the identification of the most cost-effective structure's geometry that, in general, is the one with the lowest volume of employed material. The increasing of the computational capability of the last dec- 
ades allowed the computer science to be a crucial tool in many research fields and in the optimization research field. The aim of this research work is then to outline a tool that can help the designer in the definition of the best arch geometry in the pre-design phase. To this scope in Section 5 are presented and compared some cases of optimized arch subjected to different loads.

\section{Differential Equations for the Solution of the Circular Arch}

Consider a plane curved beam segment, composed of homogeneous linear elastic material having Young modulus $E$ and with a constant curvature radius $R$, subject to the external loads $P_{t}$ and $P_{m}$, applied respectively along the tangential and normal direction, and the external distributed moment $m$. The static, kinematic and constitutive equations [22], neglecting the beam elongation $\varepsilon=0$ and the shear strain $\gamma=0$, can be combined to get the following sixth order system of differential equations that relates the external loads applied to the beam with the correspondent strains:

$$
\begin{gathered}
-E A\left(\frac{d^{2} u}{d s^{2}}\right)-G A_{S}\left(-\frac{u}{R^{2}}+\frac{1}{R} \frac{d v}{d s}-\frac{\varphi}{R}\right)=P_{t} \\
E A\left(\frac{1}{R} \frac{d u}{d s}+\frac{v}{R^{2}}\right)-G A_{S}\left(-\frac{1}{R} \frac{d u}{d s}+\frac{d^{2} v}{d s^{2}}-\frac{d \varphi}{d s}\right)=P_{n} \\
-G A_{S}\left(-\frac{u}{R}+\frac{d v}{d s}-\varphi\right)-E I \frac{d^{2} \varphi}{d s^{2}}=m
\end{gathered}
$$

where $N, V$ and $M$ the internal axial force, shear force and bending moment, $\gamma$ and $\chi$ are the elongation, shear strain and elastic curvature of the beam, while $\varphi$, $u$ and $v$ are the rotation and the tangential and normal displacements of the generic beam cross-section which the geometrical properties $A, A_{S}$ and $I$ are respectively the area, the shear-area and the bending inertia of the cross-section of the beam.

\section{Boundary Conditions and External Loads}

The method described in the Section 2 is a general method that can be applied to different load shapes and restraints. In the following one, a simplified analysis is considered based on the assumption of a fully restrained arch subject to an external distributed load constant along the arch axis $s$ and the self-weight of the structure.

The restraints can be introduced in the differential equations system by considering the boundary conditions. For a fully restrained arch, it is possible to consider null the strains in the two restrained extremities of the arch. Being $L_{\text {arch }}$ the length of the arch along the curvilinear axis $s$, the boundary conditions result:

$$
u(0)=u\left(L_{\text {arch }}\right)=0 \quad v(0)=v\left(L_{\text {arch }}\right)=0 \quad \varphi(0)=\varphi\left(L_{\text {arch }}\right)=0
$$

The external loads are given by two different contributions: the overload $q_{0}$ 
and the self-weight $q_{s w}$. The two loads are given in vertical direction and, to be applied in the differential equations system, should be projected in local system and in the directions tangential and perpendicular to the arch axis. Thus, given the self-weight as $q_{s w}=A \gamma$, it is possible to calculate $P_{t}$ and $P_{n}$ as:

$$
P_{n}=\left(\gamma A+q_{0}\right) \sin \left(\theta+\frac{s}{R}\right) \quad P_{t}=\left(\gamma A+q_{0}\right) \cos \left(\theta+\frac{s}{R}\right)
$$

\section{Numerical Solution}

The ordinary differential equations (ODEs) systems, in general, admit infinite solutions, hence it is necessary to add boundary conditions and to define a boundary value problem (BVP). The BVP is a system of ordinary differential equations subjected to a specified boundary condition in correspondence of more than one point. Therefore, the main goal is to find a solution to the ODEs which also satisfies the boundary conditions. In particular, the system defined in Section 2 is a sixth order differential equations system that can be solved by applying the sixth boundary conditions in the Equation (4). The BVP presented in the previous chapter can be solved by using the software MATLAB [19] and, in particular, the function " $b v p 4 c$ " that allows to solve problems that have two-boundary conditions, multipoint conditions, singularities in the conditions and unknown parameters. According to Shampine [18], to run the solver, the equation should be reduced to a system of first order equations, since the solvers implement a collocation method to approximate the solution of BVPs subjected to general nonlinear, two-point boundary conditions. Thus, the system formed by the three $2^{\text {nd }}$ order differential Equations (1), (2) and (3) can be rewritten as system of formed by six $1^{\text {st }}$ order differential equations by defining the first derivatives of the strain as unknowns of the system:

$$
u_{1}=u \quad v_{1}=v \quad \varphi_{1}=\varphi u_{2}=\frac{d u}{d s} \quad v_{2}=\frac{d v}{d s} \quad \varphi_{2}=\frac{d \varphi}{d s}
$$

Under the previous assumptions, it is possible to rewrite the sixth order differential equations system as follows:

$$
\left\{\begin{array}{l}
\frac{d u_{1}}{d s} \\
\frac{d v_{1}}{d s} \\
\frac{d \varphi_{1}}{d s} \\
\frac{d u_{2}}{d s} \\
\frac{d v_{2}}{d s} \\
\frac{d \varphi_{2}}{d s}
\end{array}\right\}=\left[\begin{array}{cccccc}
0 & 0 & 0 & 1 & 0 & 0 \\
0 & 0 & 0 & 0 & 1 & 0 \\
0 & 0 & 0 & 0 & 0 & 1 \\
\frac{D}{R^{2}} & 0 & \frac{D}{R} & 0 & -\frac{D+1}{R} & 0 \\
0 & \frac{1}{D R^{2}} & 0 & \frac{1}{D R}+\frac{1}{R} & 0 & 1 \\
\frac{D^{I}}{R} & 0 & D^{I} & 1 & -D^{I} & 0
\end{array}\right]\left\{\begin{array}{c}
u_{1} \\
v_{1} \\
\varphi_{1} \\
u_{2} \\
v_{2} \\
\varphi_{2}
\end{array}\right\}+\left\{\begin{array}{c}
0 \\
0 \\
0 \\
-\frac{P_{t}}{E A} \\
-\frac{P_{n}}{E I} \\
0
\end{array}\right\}
$$

where $D=G A_{S} \backslash E A$ and $D^{I}=G A_{S} \backslash E I$. Moreover the arch was considered 
fully fixed at each end as in Figure 1 for this reason the boundary conditions can be imposed as in the Equations (4). The calculation method presented in this paper has been validated by comparing the obtained results in MATLAB [19] with the results obtained by building a finite element equivalent model in the software MidasGen [23]. In particular, the results are compared in terms of internal actions (Figure 2), displacements calculated in the global coordinate system $(x, y)$ (Figure 3), rotations (Figure 4) and combined compressive and bending

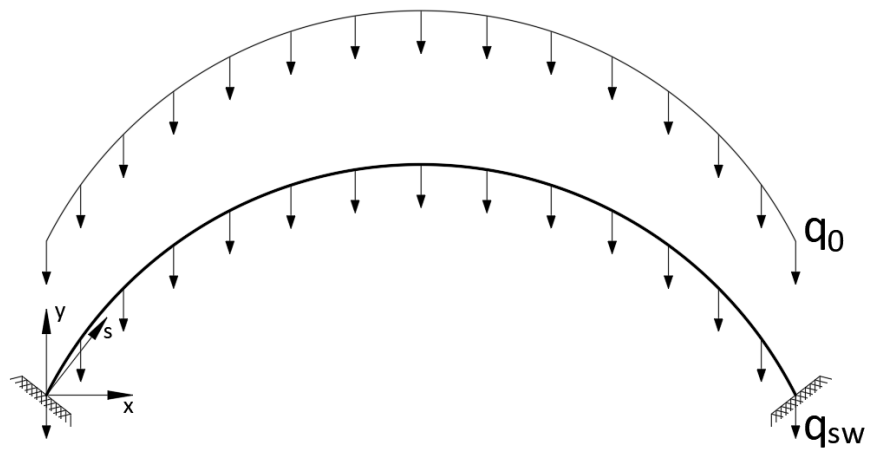

Figure 1. Restraints and external load acting on the arch.

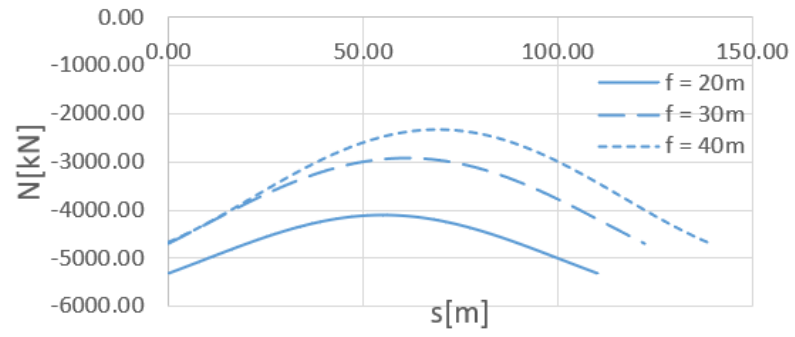

(a)

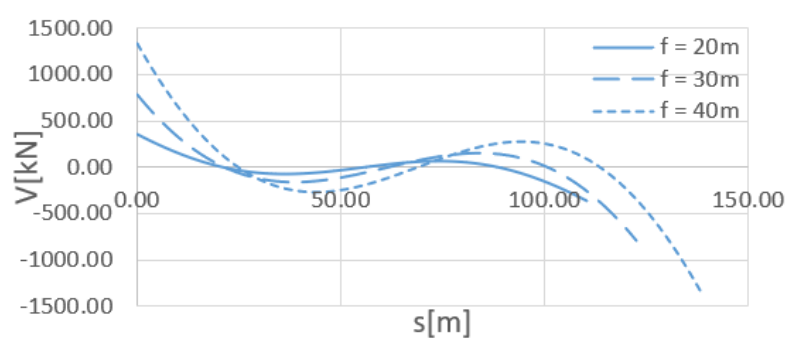

(c)

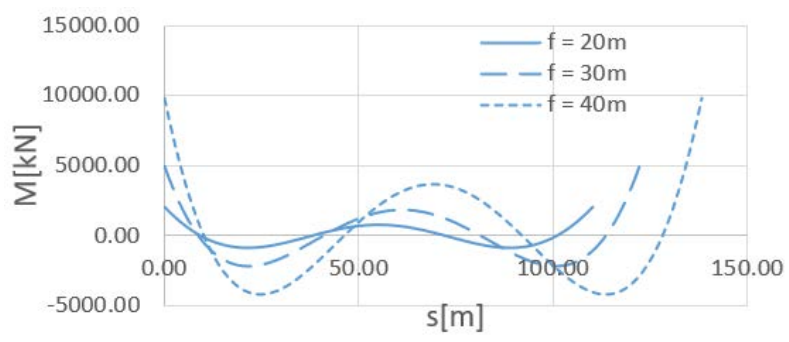

(e)

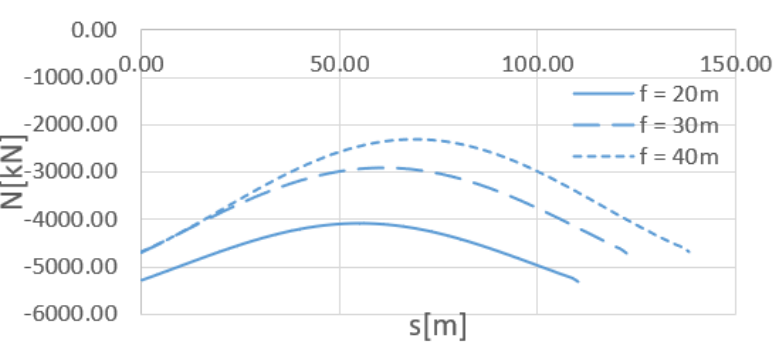

(b)

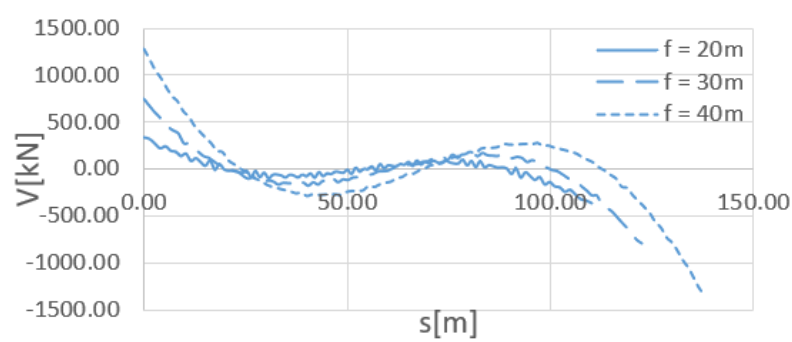

(d)

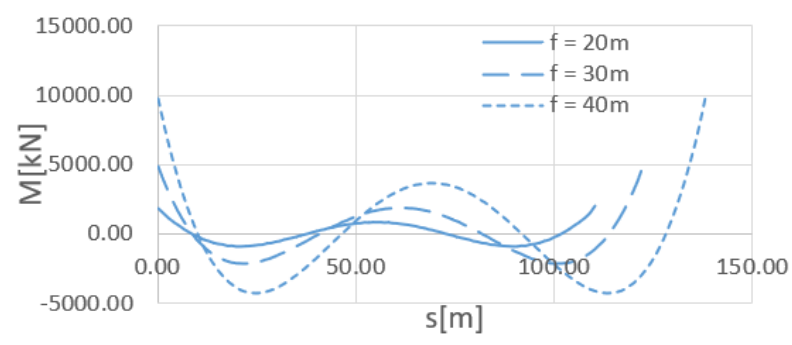

(f)

Figure 2. Comparison of Internal forces: Axial Force (MATLAB (a) and Midas (b)), Shear Force (MATLAB (c) and Midas (d)), Bending Moment (MATLAB (e) and Midas (f)). 


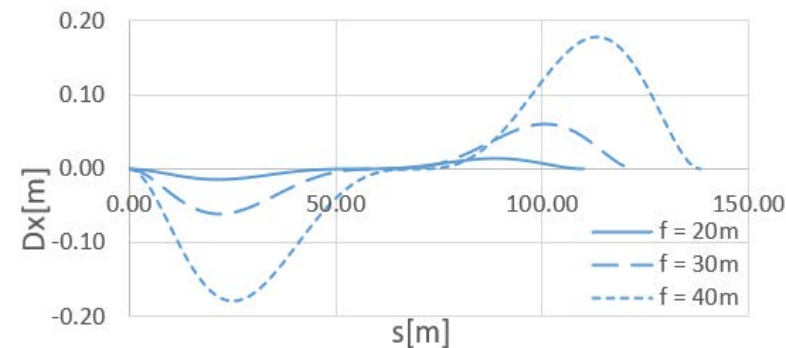

(a)

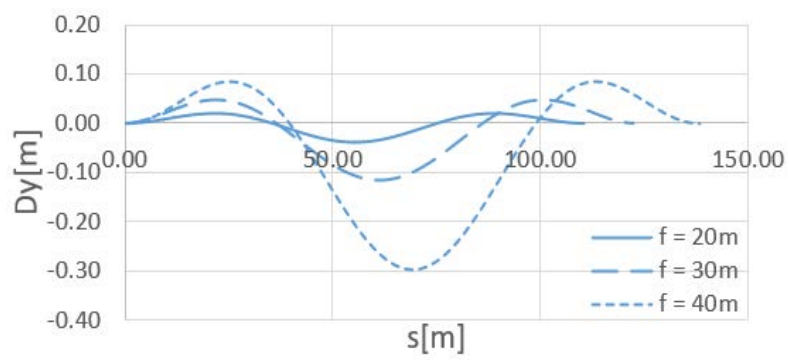

(c)

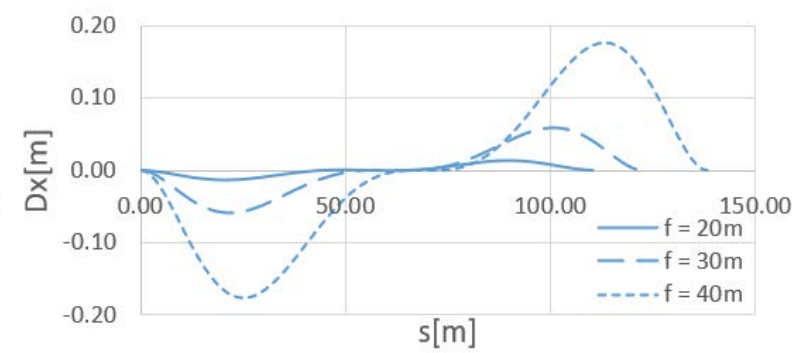

(b)

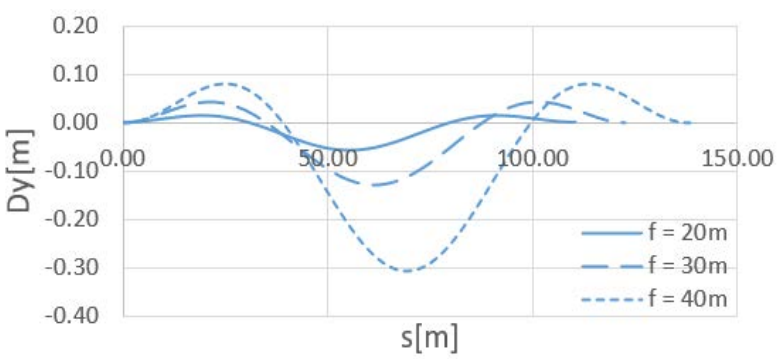

(d)

Figure 3. Comparison of the Displacements in the global coordinate system $(x, y)$ : Displacements in the $x$-direction (MATLAB (a) and Midas (b)), Displacements in the y-direction (MATLAB (c) and Midas (d)).

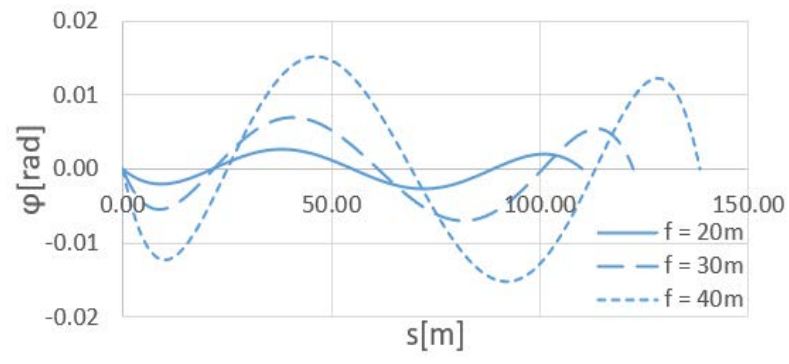

(a)

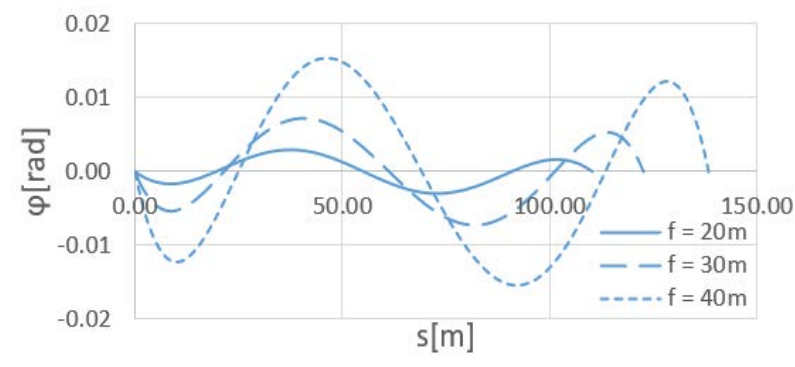

(b)

Figure 4. Comparison of the Rotations (MATLAB (a) and Midas (b)).

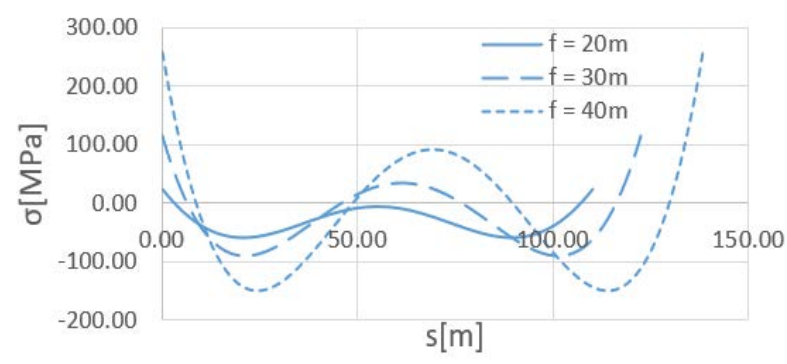

(a)

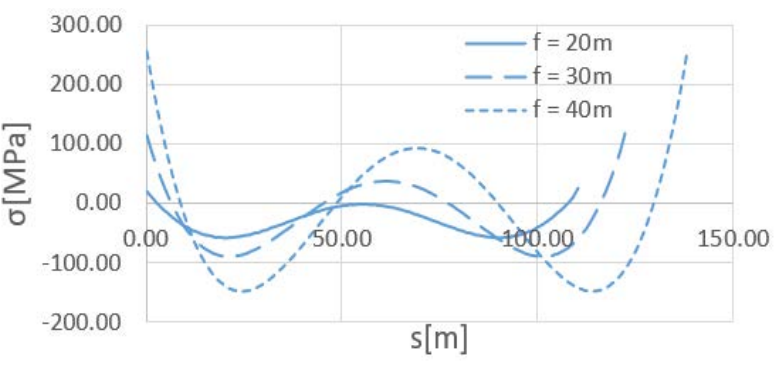

(b)

Figure 5. Comparison of the Normal stresses (MATLAB (a), and Midas (b)).

stress (Figure 5). In the following, it is reported the example of the results comparison for some steel arches characterized by a span $L=100 \mathrm{~m}$ and hollow circular cross-section with a radius $r=0.50 \mathrm{~m}$ and a thickness $t=0.05 \mathrm{~m}$. The arches are subjected to an external load $q_{0}=-50 \mathrm{kN} / \mathrm{mZ}$ and the results are calculated for different height in the midspan $f=[10,20,30,40] \mathrm{m}$. 


\section{Optimization and Genetic Algorithm}

The aim of this paper is to propose a tool useful in the pre-design phase for the definition of the arch geometry, able to bear the design loads and, at the same time, that requires as less material as possible. In particular, the selected optimization algorithm is the genetic one that, according to the MATLAB User Guide [19] and Goldberg [20] [21], is a machine learning algorithm based on a stochastic optimization method for solving both constrained and unconstrained optimization problems. The method is based on the mechanics of natural selection and natural genetics, process that drives biological evolution [24]. The genetic algorithm iteratively modifies a population of individual solutions: at each step, it selects individuals at random from the current population to be parents, used to produce the "children" for the next generation. Over successive generations, the population "evolves" toward an optimal solution [25]. The algorithm requires the definition of the variables, the objective function and the constraints. In this analysis it is defined a vector of variables that is used to define the geometry of the arch, the first variable is the angle $\theta$ (in Figure 7) that defines the shape of the arch while the others are the dimensions of the cross-section. For simplicity it is decided to restrict the field of the analysis to cross-sections that can be fully defined by two parameters at most, in such a way that the variables to be optimized will be no more than 3 . The selected optimization objective function is the volume of the arch. It is proportional to the amount of material and has to be calculated at each iteration as a function of the design variables until the geometry corresponding to the minimum volume is found. Finally, the constraint of the optimization problem is represented by the maximum normal stresses that arise on the arch structure due to the application of the loads. The algorithm must verify that the value of the maximum stress applied $\sigma_{\max }$ is less than the maximum yield strength of the material that constitutes the arch $f_{y}$. A simplified analysis is chosen that considers only the stresses perpendicular to the cross-section and that are given by the combination of the axial force and the bending moment. The shear stresses are not considered. Moreover, the analysis is performed without accounting for the instability effects [26], but being the method quite general it is possible to add such constraints in further developments. The algorithm has been tested by optimizing an arch with a span $L=100 \mathrm{~m}$ made of a structural steel S355 with a yield strength $f_{y}=355 \mathrm{MPa}$. The arch has been optimized by applying different external loads $q_{0}=[-250,-350,-450,-550,-650] \mathrm{kN} / \mathrm{m}$. Initially the analysis has been performed by considering a filled circular cross-section with a number of design variables $n_{v a r}=2$. The first variable $\theta$, defined in Figure 6 , is the angle between the line that connect the arch center to the restraint at the base and the horizontal plane; this angle fully describes the geometry of the arch. The second variable is represented by the radius of the cross-section $r$ as in Figure 7.

Once that the optimization is performed for the different load amounts, it has been checked the effectiveness of the algorithm by plotting in the same graph 
both the stresses acting on the optimized arches and both the yield strength of the used steel $f_{y}$.

In Figure 8 all the curves that represent the maximum acting stress $\sigma_{\max }$ are tangent to the lines that stay for $f_{y}$. The tangency can be interpreted as the proof that optimization is effective because means that the optimized configurations are the ones that require a quantity of material that is just sufficient to bear the design loads. The second test of the algorithm has been performed by considering the same span length $L$, the same design loads $q_{0}$ and the same material of the previous case, but different cross-section, considering a filled ellipsoidal

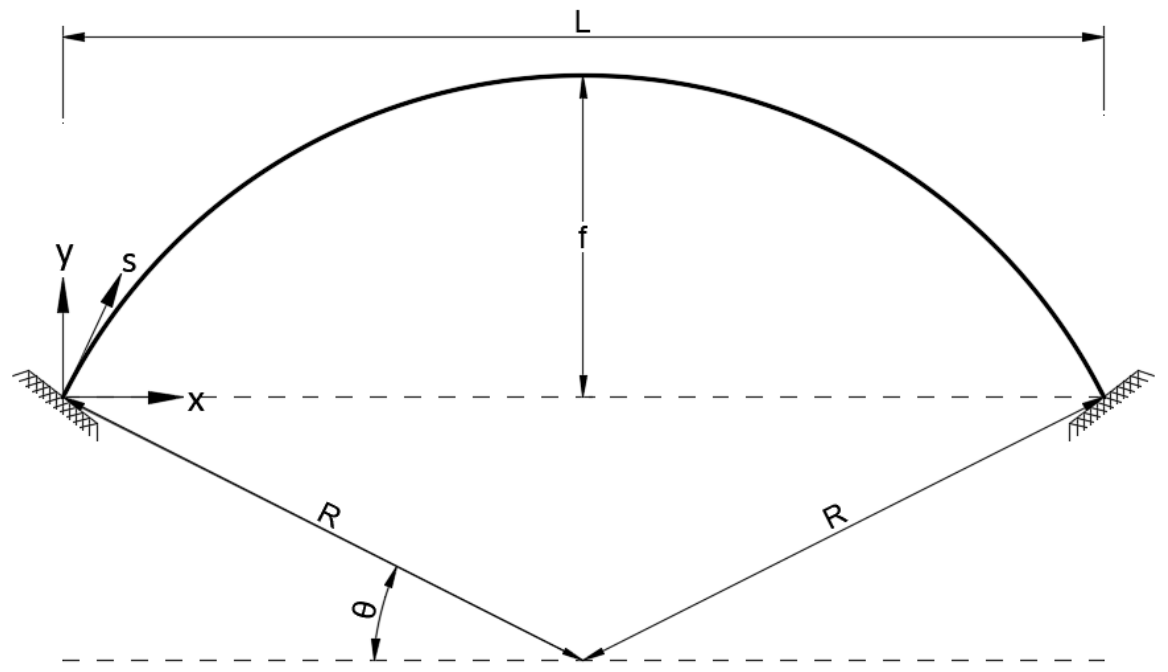

Figure 6. Geometrical parameters of the arch.

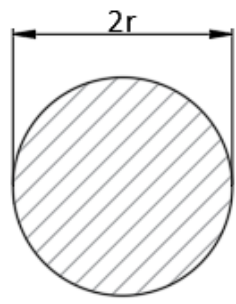

Filled Circular

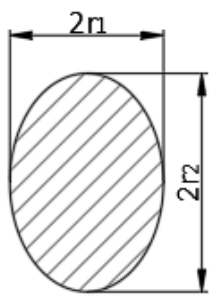

Filled Ellipsoidal

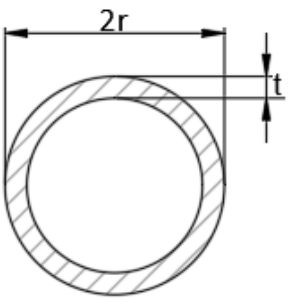

Hollow circular

Figure 7. Geometrical parameters of the cross-sections.

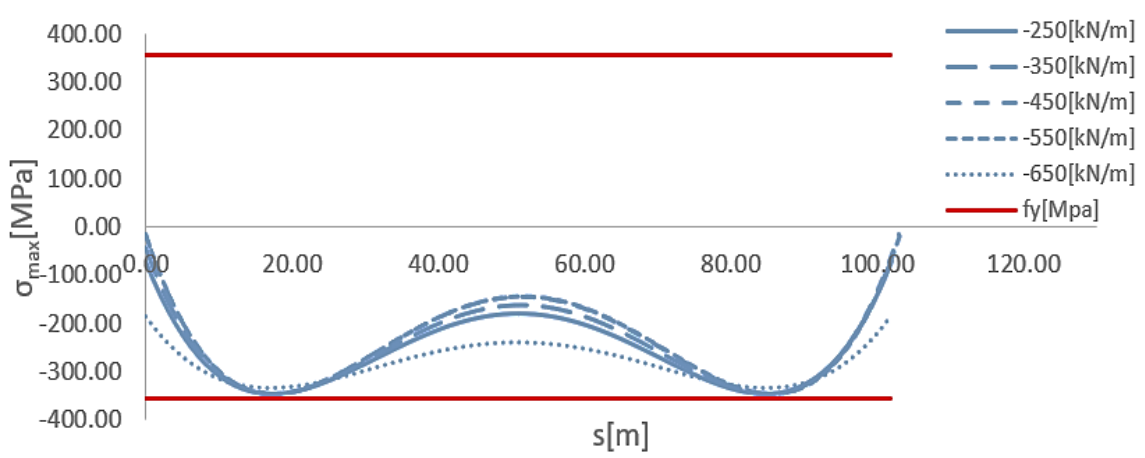

Figure 8. Filled circular cross-section: Comparison between the stresses on the optimized arches subjected to the design loads $\sigma_{\max }$ and the yield stress of the material $f_{y}$. 
one to test the algorithm with $n_{v a r}=3$. The first design variable is the angle $\theta$ used to define the geometry of the arch. The second and third variables represent the dimensions of the cross-section as for the ellipse represented in Figure 7. Even in this case, it has been compared the acting normal stresses with the yield strength of the material and, as in the previous case, it is possible to notice in Figure 9 how the acting stress curves are tangent to the yield strength limit.

The last optimization example refers to the same arch of the previous two case studies but with a hollow circular cross-section. Even in this case, the algorithm is tested with a number of design variables $n_{v a r}=3$ but the idea is to use a steel cross-section that has the capability of develop a higher inertia by using the same amount of material. The first design variable is the angle $\theta$, while the second and third variables are respectively the external radius and the thickness of the cross-section, as represented in Figure 7. In Figure 10, it is possible to observe the same behavior of the maximum acting stress curves already observed in the previous case studies.

Finally, Figure 11 shows how the volume of materials that constitute the optimized arch configurations varies as a function of the applied design loads. It can be observed how, increasing the acting load, the quantity of material will increase. Furthermore, it is possible to compare the volume of material required to carry the same applied loads with different arch's cross-section. It can be noticed that the configuration with 3 design variables is more efficient than the one

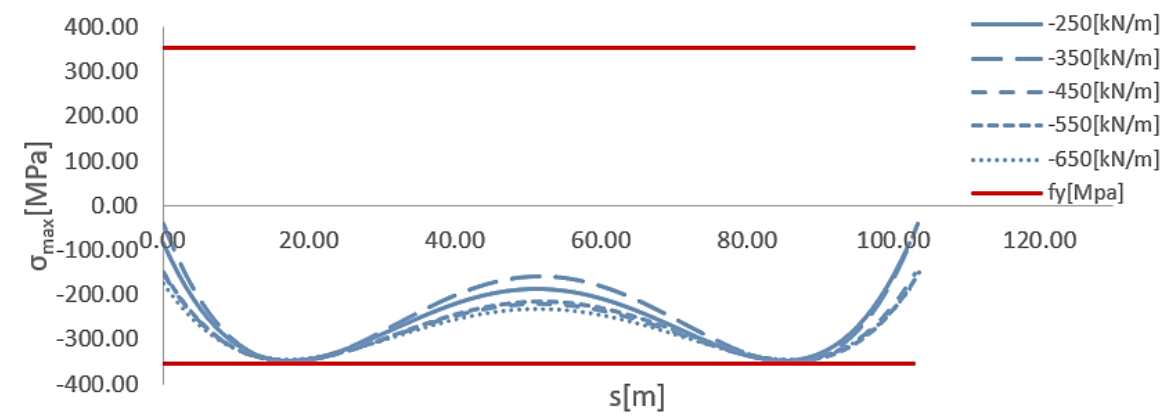

Figure 9. Filled ellipsoidal cross-section: Comparison between the stresses on the optimized arches subjected to the design loads $\sigma_{\max }$ and the yield stress of the material $f_{y}$.

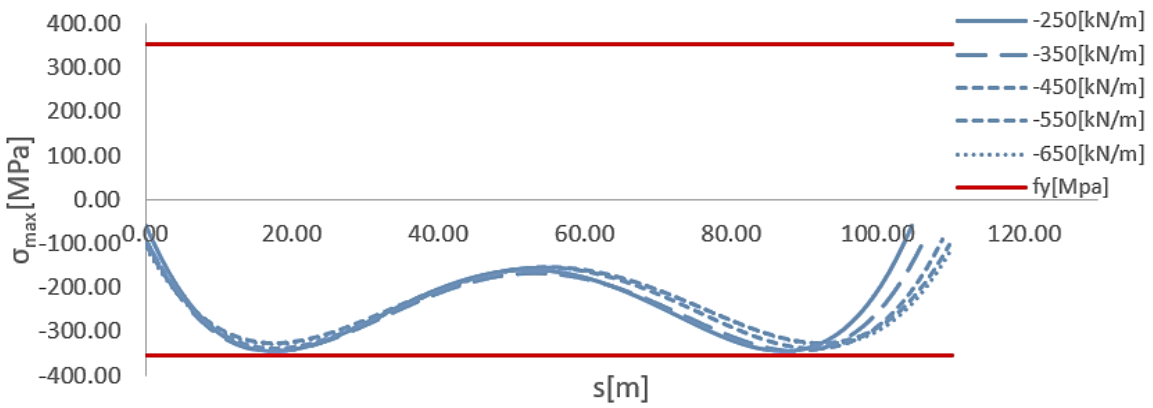

Figure 10. Hollow circular cross-section: Comparison between the stresses on the optimized arches subjected to the design loads $\sigma_{\max }$ and the yield stress of the material $f_{y}$. 


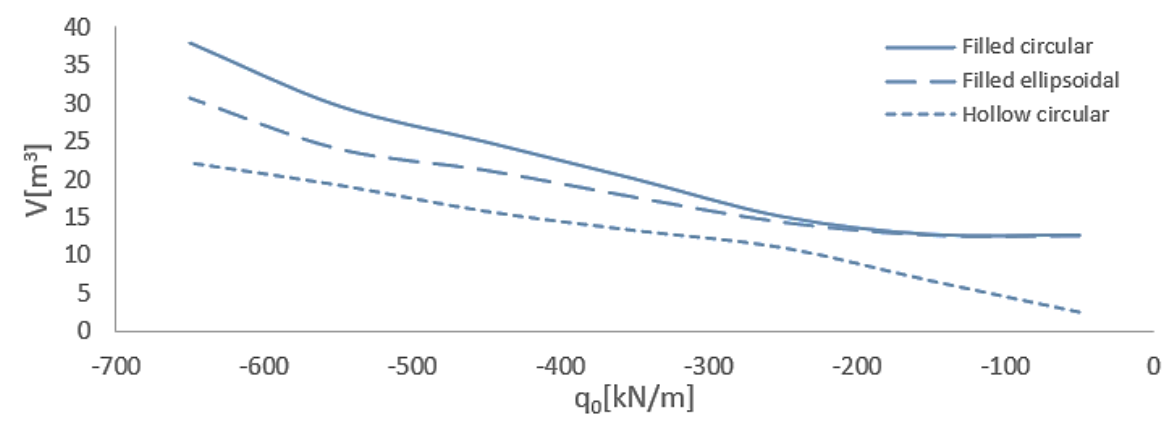

Figure 11. Comparison of the volumes of material required by the different arch configurations to bear the design loads.

characterized by just twos. This is quite normal as, having more variables, the algorithm can optimize one more geometrical quantity and thus will result in a more efficient configuration. It can also be noticed that the arch with the hollow cross-section is the one that requires the less volume of material as these types of steel cross-section are universally recognized as more efficient with respect to the filled ones.

\section{Conclusion}

An analytical-numerical formulation and a MATLAB [19] based solution procedure is here proposed for the calculation of the internal stresses for circular arches subjected to distributed loads combined with their own weight. The formulation is based on the solution of a sixth order differential equation system through the boundary value problem solver algorithm " $b v p 4 c$ " implemented in the software MATLAB [19]. The solution of the equations system allows the calculation of the arch displacement and consequently of the internal actions and stresses. The formulation and the method are validated by the comparison of the results computed for different case studies with the ones obtained with a commercial FEM software. The method is, then, implemented as a constraint function in the genetic algorithm that is used to obtain the optimal geometrical parameters of arches with different cross-sections and subject to different loading configurations. The results have been plotted together with the yield strength of the material to verify the effectiveness of the optimization algorithm. It has been studied the cases of filled circular, filled ellipsoidal and hollow circular cross-sections. The aim of this choice was to compare the different degree of optimization that can be achieved with different geometries and shapes. The filled circular case study is the simplest one, since a single parameter to fully describe the cross-section geometry was sufficient, while the case of ellipsoidal cross-section has been chosen because two parameters are necessary to the definition of the cross-section geometry. Moreover, the case of hollow circular cross-section was investigated, because, as the ellipsoidal one, in this case, two parameters are necessary to define the cross-section geometry and, in addition, because the hollow steel cross-sections are very used in civil constructions and it is universally considered very efficient. Also in the simulations, here proposed, the confirmation 
of the validity of the last section typology lies in the fact that the volumes of optimized arches, with this cross-section, are the lowest ones. Finally, the results reported in the present paper represented a valid application of artificial intelligence algorithms for the structural optimization of circular arches.

\section{Conflicts of Interest}

The authors declare no conflicts of interest regarding the publication of this paper.

\section{References}

[1] Jasińska, D. and Kropiowska, D. (2018) The Optimal Design of an Arch Girder of Variable Curvature and Stiffness by Means of Control Theory. Mathematical Problems in Engineering, 2018, Article ID: 8239464.

https://doi.org/10.1155/2018/8239464

[2] Szefer, G. and Mikulski, L. (1984) Optimal Design of Elastic Arches with I CrossSection. Engineering Transactions, 32, 467-480.

[3] Marano, G.C., Trentadue, F. and Petrone, F. (2014) Optimal Arch Shape Solution under Static Vertical Loads. Acta Mechanica, 225, 679-686. https://doi.org/10.1007/s00707-013-0985-0

[4] Trentadue, F., Marano, G.C., Vanzi, I. and Briseghella, B. (2018) Optimal Arches Shape for Single-Point-Supported Deck Bridges. Acta Mechanica, 229, 2291-2297. https://doi.org/10.1007/s00707-017-2084-0

[5] Farshad, M. (1976) On Optimal Form of Arches. Journal of the Franklin Institute, 302, 187-194. https://doi.org/10.1016/0016-0032(76)90022-3

[6] Kimura, T., Ohsaki, M., Fujita, S., Michiels, T. and Adriaenssens, S. (2020) Shape Optimization of No-Tension Arches Subjected to In-Plane Loading. Structures, 28, 158-169. https://doi.org/10.1016/j.istruc.2020.08.053

[7] Hu, C., Wan, Y. and ShangGuan, X. (2010) A New Practice in the Design of Arch Axis. Proceedings of the 6th International Conference on Arch Bridges, Fuzhou, 709-715.

[8] Osserman, R. (2010) How the Gateway Arch Got Its Shape. Nexus Network Journal, 12, 167-189. https://doi.org/10.1007/s00004-010-0030-8

[9] Michiels, T. and Adriaenssens, S. (2018) Form-Finding Algorithm for Masonry Arches Subjected to In-Plane Earthquake Loading. Computers \& Structures, 195, 85-98. https://doi.org/10.1016/j.compstruc.2017.10.001

[10] Halpern, A.B. and Adriaenssens, S. (2015) In-Plane Optimization of Truss Arch Footbridges Using Stability and Serviceability Objective Functions. Structural and Multidisciplinary Optimization, 51, 971-985. https://doi.org/10.1007/s00158-014-1187-7

[11] Marmo, F. (2021) ArchLab: A MATLAB Tool for the Thrust Line Analysis of Masonry Arches. Curved and Layer. Struct., 8, 26-35. https://doi.org/10.1515/cls-2021-0003

[12] Manuello, A. (2020) Multi-Body Rope Approach for Grid Shells: Form-Finding and Imperfection Sensitivity. Engineering Structures, 221, Article ID: 111029. https://doi.org/10.1016/j.engstruct.2020.111029

[13] Houšt, V., Eliáš, J. and Miča, L. (2013) Shape Optimization of Concrete Buried Arches. Engineering Structures, 48, 716-726. 
https://doi.org/10.1016/j.engstruct.2012.11.037

[14] Habbal, A. (1998) Direct Approach to the Minimization of the Maximal Stress over an Arch Structure. Journal of Optimization Theory and Applications, 97, 551-578. https://doi.org/10.1023/A:1022685908429

[15] Kumarci, K., Dehkordy, P.K., Mahmodi, I., et al. (2009) Optimum Shape in Brick Masonry Arches under Dynamic Loads by Cellular Automata. Journal of Civil Engineering, 37, 73-90.

[16] Park, J., Chun, Y.-H. and Lee, J. (2016) Optimal Design of an Arch Bridge with High Performance Steel for Bridges Using Genetic Algorithm. International Journal of Steel Structures, 16, 559-572. https://doi.org/10.1007/s13296-016-6024-y

[17] Pouraminian, M. and Ghaemian, M. (2015) Shape Optimisation of Concrete Open Spandrel Arch Bridges. Gradjevinar, 67, 1177-1185.

[18] Shampine, L.F., Kierzenka, J. and Reichelt, J.M.W. (2000) Solving Boundary Value Problems for Ordinary Differential Equations in MATLAB with bvp4c. Tutorial notes, 1-27.

[19] https://www.mathworks.com/products/matlab.html

[20] Goldberg, D.E. and Holland, J.H. (1988) Genetic Algorithms and Machine Learning. Kluwer Academic Publishers, Manufactured in The Netherlands.

[21] Goldberg, D.E. (1989) Genetic Algorithms in Search, Optimization, and Machine Learning. Addison-Wesley, Reading.

[22] Carpinteri, A. (2013) Structural Mechanics Fundamentals. CRC Press Taylor and Francis Group. https://doi.org/10.1201/9781315272528

[23] https://www.midasoft.com/building/products/midasgen

[24] Holland, J.H. (1992) Genetic Algorithms. Scientific American, 267, 66-73. https://doi.org/10.1038/scientificamerican0792-66

[25] Grefenstette, J.J. (1989) How Genetic Algorithms Work: A Critical Look at Implicit Parallelism. Proceedings of the 3 rd International Joint Conference on Genetic Algorithms (ICGA89).

[26] Bazzucchi, F., Manuello, A. and Carpinteri, A. (2017) Interaction between SnapThrough and Eulerian Instability in Shallow Structures. International Journal of Non-Linear Mechanics, 88, 11-20.

https://doi.org/10.1016/j.ijnonlinmec.2016.10.006 